\title{
Decorated $\mathbb{Z}_{2}$ symmetry defects and their time-reversal anomalies
}

\author{
Clay Córdova, ${ }^{1,2, *}$ Kantaro Ohmori, ${ }^{3,2, \dagger}$ Shu-Heng Shao ${ }^{2,+}$ and Fei Yan $\oplus^{4, \S}$ \\ ${ }^{1}$ Kadanoff Center for Theoretical Physics \& Enrico Fermi Institute, University of Chicago, \\ Chicago, Illinois 60637, USA \\ ${ }^{2}$ School of Natural Sciences, Institute for Advanced Study, Princeton, New Jersey 08540, USA \\ ${ }^{3}$ Simons Center for Geometry and Physics, State University of New York, \\ Stony Brook, New York 11794, USA \\ ${ }^{4}$ NHETC and Department of Physics and Astronomy, Rutgers University, \\ Piscataway, New Jersey 08854, USA
}

(Received 22 November 2019; accepted 10 August 2020; published 27 August 2020)

\begin{abstract}
We discuss an isomorphism between the possible anomalies of $(d+1)$-dimensional quantum field theories with $\mathbb{Z}_{2}$ unitary global symmetry, and those of $d$-dimensional quantum field theories with timereversal symmetry $T$. This correspondence is an instance of symmetry defect decoration. The worldvolume of a $\mathbb{Z}_{2}$ symmetry defect is naturally invariant under $T$, and bulk $\mathbb{Z}_{2}$ anomalies descend to $T$ anomalies on these defects. We illustrate this correspondence in detail for $(1+1) d$ bosonic systems where the bulk $\mathbb{Z}_{2}$ anomaly leads to a Kramers degeneracy in the symmetry defect Hilbert space and exhibits examples. We also discuss $(1+1) d$ fermion systems protected by $\mathbb{Z}_{2}$ global symmetry where interactions lead to a $\mathbb{Z}_{8}$ classification of anomalies. Under the correspondence, this is directly related to the $\mathbb{Z}_{8}$ classification of $(0+1) d$ fermions protected by $T$. Finally, we consider $(3+1) d$ bosonic systems with $\mathbb{Z}_{2}$ symmetry where the possible anomalies are classified by $\mathbb{Z}_{2} \times \mathbb{Z}_{2}$. We construct topological field theories realizing these anomalies and show that their associated symmetry defects support anyons that can be either fermions or Kramers doublets.
\end{abstract}

DOI: 10.1103/PhysRevD.102.045019

\section{INTRODUCTION}

Global symmetries and anomalies are crucial tools in the analysis of quantum field theory. In its most elementary incarnation, global symmetry organizes Hilbert spaces into representations and gives rise to selection rules. Anomalies are more subtle characteristic invariants of symmetry in quantum field theory and are constant along all continuous symmetry-preserving deformations of the theory, including scaling transformations of the renormalization group. A rich class of phenomena are associated with systems with discrete global symmetry. In this case the associated anomalies are also finite order and can be carried by gapped or gapless systems. A precise understanding of the physics of anomalies is a central tool in many recent developments including the theory of topological insulators

\footnotetext{
*clayc@uchicago.edu

†omori@scgp.stonybrook.edu

shao@ias.edu

${ }^{\S}$ fei.yan@physics.rutgers.edu
}

Published by the American Physical Society under the terms of the Creative Commons Attribution 4.0 International license. Further distribution of this work must maintain attribution to the author(s) and the published article's title, journal citation, and DOI. Funded by SCOAP ${ }^{3}$. and superconductors, duality in $(2+1) d$, and topological field theory.

In this paper, we discuss an interesting connection between anomalies of $\mathbb{Z}_{2}$ unitary global symmetry in $d$ spacetime dimensions, and anomalies for antiunitary timereversal $(\mathrm{T})$ symmetries in $(d-1)$ spacetime dimensions. Since anomalies of $d$ dimensional theories are determined by inflow from $(d+1)$-dimensional symmetry-protected topological phases (SPTs), our discussion can also be interpreted as a connection between SPTs. Specifically, there is an isomorphism:

$$
\begin{aligned}
& \mathbb{Z}_{2} \text { anomalies in } d \text { dimensions } \\
& \quad \leftrightarrow \mathrm{T} \text { anomalies in }(d-1) \text { dimensions. }
\end{aligned}
$$

Mathematically, SPT phases (including interactions) are classified by cobordism theory [1-8]. The precise mathematical relationship behind (1.1) is sometimes referred to as a Smith isomorphism $[9,10]$ (see also $[4,11]$ ), which we review in Appendix.

Our main goal in this paper is to explore the physical consequences of (1.1) in low-dimensional field theory examples. In particular, we illustrate this correspondence in a simple class of models described by $(1+1) d$ bosonic quantum field theories (QFTs) with $\mathbb{Z}_{2}$ global symmetry. We 


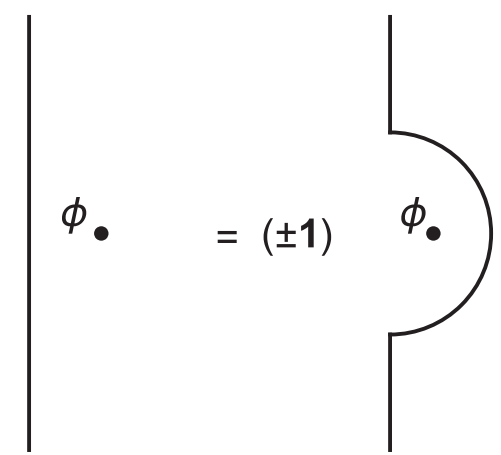

FIG. 1. The action of a $\mathbb{Z}_{2}$ symmetry defect. The defect is topological and small changes in its position do not modify correlation functions. However, when the defect crosses a local operator $\phi$, the correlation changes by \pm 1 depending on whether $\phi$ is even or odd under the action of the symmetry.

argue that this symmetry is anomalous if and only if the $(0+1) d$ symmetry line has states that are related by a Kramers degeneracy. We also discuss the extension to $(1+1) d$ fermionic systems and give an example application in $(3+1) d$ bosonic systems.

\section{A. $\mathbb{Z}_{2}$ symmetry defects and time-reversal symmetry}

Physically, the isomorphism (1.1) is mediated by symmetry defects. In general in QFT, the abstract meaning of the symmetry, illustrated in Fig. 1, is that there are codimension one topological operators that implement the $\mathbb{Z}_{2}$ symmetry action (see, e.g., [12]). Equivalently one can view the presence of symmetry defects in correlation functions as coupling the QFT to a background $\mathbb{Z}_{2}$ gauge field. ${ }^{1}$

The key observation behind the isomorphism (1.1) is then that in a $d$-dimensional QFT with $\mathbb{Z}_{2}$ global symmetry, the symmetry defect is a $(d-1)$-dimensional quantum system with T symmetry. This can be understood geometrically. The analog of a symmetry defect for $T$ is a locus where the orientation of spacetime is reversed. Equivalently, this means that a QFT has T symmetry if it can be formulated on nonorientable manifolds. In general, symmetry defects are defined on oriented manifolds, and changing the orientation leads to a defect for the inverse symmetry group element. However, in the special case of $\mathbb{Z}_{2}$, the nontrivial group element is its own inverse and hence the defect is invariant under changing its orientation. As argued above, this means that the defect theory has $\mathrm{T}$ symmetry. (For more details see Appendix.)

\footnotetext{
${ }^{1}$ Mathematically, such a gauge field is a cocycle $a \in Z^{1}\left(M, \mathbb{Z}_{2}\right)$, where $M$ is the $d$-dimensional spacetime. The symmetry defect is then Poincare dual to the cocycle $a$. The associated gauge equivalence class is $[a] \in H^{1}\left(M, \mathbb{Z}_{2}\right)$.
}

Another useful point of view can be obtained in the phase where the $\mathbb{Z}_{2}$ symmetry is spontaneously broken, illustrated in Fig. 2. In that case the $\mathbb{Z}_{2}$ symmetry defect is realized as a domain wall separating two distinct vacua. A global $\mathbb{Z}_{2}$ transformation does not preserve this configuration since it exchanges the vacua. However, if we combine this $\mathbb{Z}_{2}$ with the universal CPT symmetry, which acts in the Euclidean signature as a rotation by $\pi$, we indeed find a symmetry of the original domain wall configuration. As this combined action reverses the orientation of the $\mathbb{Z}_{2}$ symmetry defect, it descends to a $T$ symmetry on its worldvolume.

Thus, given any QFT with unitary $\mathbb{Z}_{2}$ global symmetry, the symmetry defect yields a theory in one lower dimension with antiunitary $\mathrm{T}$ symmetry. Moreover, if the latter is anomalous, necessarily so is the former. In fact, because (1.1) is an isomorphism, all nontrivial $\mathbb{Z}_{2}$ anomalies of the bulk can be detected in this way. Using inflow, the same arguments apply to the associated SPTs characterizing the anomalies.

The idea that the worldvolume theory of symmetry defects can be used to encode bulk anomalies is widely applied in the condensed matter literature [13]. Here we see that the isomorphism (1.1) gives an instance of this idea, where the $\mathbb{Z}_{2}$ symmetry defects are decorated theories with anomalous $\mathrm{T}$ symmetry. The novelty, however, is that the T symmetry on the defect is not a symmetry of the bulk.

One sharp way to realize the results of this correspondence is by circle compactification. Consider a $d$-dimensional theory on a geometry $S^{1} \times \mathcal{L}$ for some $(d-1)$ manifold $\mathcal{L}$, and let a $\mathbb{Z}_{2}$ symmetry defect wrap $\mathcal{L}$ (and hence be located at a point in the $S^{1}$ ). Equivalently, this means that there is a nontrivial $\mathbb{Z}_{2}$ holonomy around the $S^{1}$. We can describe this configuration as an effective $(d-1)$-dimensional QFT. According to the analysis above, this QFT has T symmetry, with a $T$ anomaly that completely encodes the original $\mathbb{Z}_{2}$ anomaly of the $d$-dimensional theory. In particular, thinking of $\mathcal{L}$ as extended along time, this means that the defect Hilbert space $\mathcal{H}_{\mathcal{L}}$ of the original theory (again with $\mathcal{L}$ localized at a point in a spatial circle) carries $\mathrm{T}$ symmetry.

\section{B. Implications for $(1+1) d$ QFT}

While the analysis above (and its generalization to spin theories discussed below) apply in arbitrary spacetime dimension, one focus of this paper is to understand in detail the map between $(1+1) d$ theories with $\mathbb{Z}_{2}$ symmetry, and quantum mechanics models with $\mathrm{T}$ symmetry.

In the $(1+1) d$ case, the geometry of the symmetry defects and the map (1.1) is particularly simple. The $\mathbb{Z}_{2}$ symmetry is represented by a topological line $\mathcal{L}$ in the theory. The general properties of these lines have been analyzed in [14-23]. For (bosonic) $(1+1) d$ QFTs with $\mathbb{Z}_{2}$ symmetry there is a unique possible nontrivial 't Hooft 


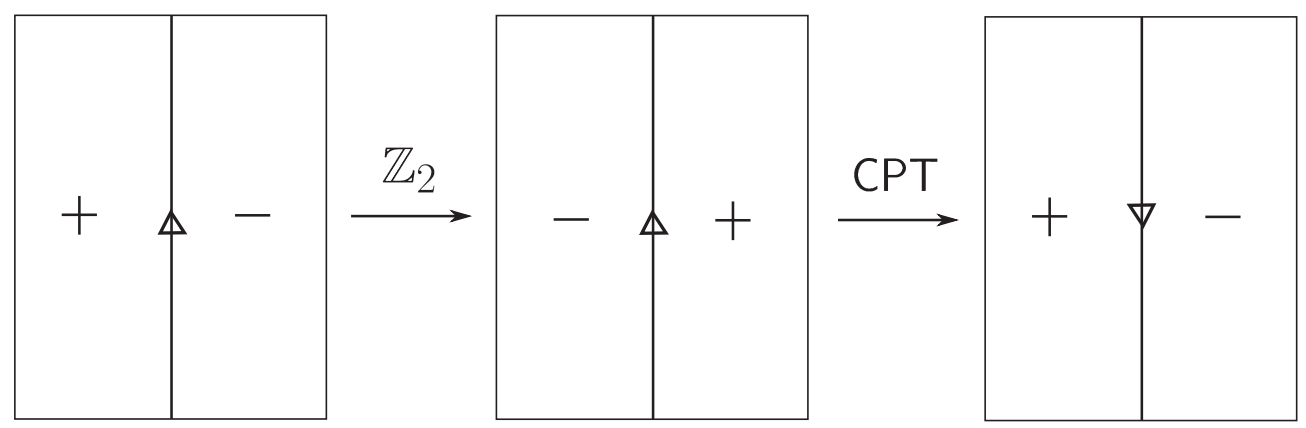

FIG. 2. The T symmetry on the symmetry defect in a spontaneously broken phase. The defect separates two regions with degenerate ground states labeled by \pm . The action of the $\mathbb{Z}_{2}$ symmetry exchanges the vacua, and combining with CPT leads to a symmetry of the original configuration that changes the worldvolume orientation of the defect.

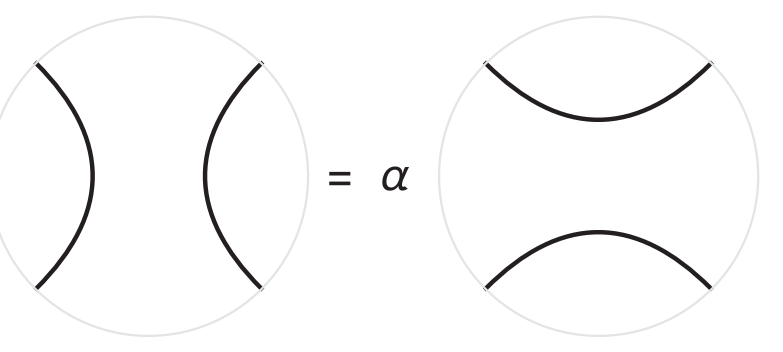

FIG. 3. The crossing relation of a $\mathbb{Z}_{2}$ line $\mathcal{L}$ (shown in black) in a local patch of the $(1+1) d$ geometry. The lines can be recombined at the cost of a phase $\alpha= \pm 1$. The $\mathbb{Z}_{2}$ symmetry is anomalous (i.e., there is an obstruction to orbifolding), if and only if $\alpha=-1$.

anomaly. ${ }^{2}$ Following the general analysis in [22-24], it is easy to realize that this anomaly controls the crossing relation of the symmetry defects illustrated in Fig. 3.

We use these results to independently argue that the line $\mathcal{L}$ has a $T$ symmetry which is anomalous if and only if the $(1+1) d$ theory has an anomalous $\mathbb{Z}_{2}$ symmetry. Since $\mathcal{L}$ is one-dimensional, this is an anomaly in quantum mechanics, i.e., a projective representation of the global symmetry. For $\mathrm{T}$ symmetry this means that the system has an anomaly if and only if, on the Hilbert space

$$
\mathrm{T}^{2}=-1
$$

In other words, the Hilbert space consists of Kramers doublets. We directly investigate defect Hilbert spaces, $\mathcal{H}_{\mathcal{L}}$

${ }^{2}$ This anomaly is characterized by inflow from the $(2+1) d$ SPT with classical action

$$
\exp \left(\pi i \int_{N} a \cup a \cup a\right)
$$

where again $a \in H^{1}\left(N, \mathbb{Z}_{2}\right)$ is the class representing the $\mathbb{Z}_{2}$ gauge field. Alternatively, we can view this SPT as characterizing the nontrivial element in the group cohomology, $H^{3}\left(\mathbb{Z}_{2}, U(1)\right) \cong \mathbb{Z}_{2}$. in a variety of examples of $(1+1) d$ conformal field theories (CFTs), and verify this conclusion.

\section{Generalization to spin systems}

Although much of our discussion concerns bosonic theories. The correspondence (1.1) also holds for fermionic systems. In this case, our system also has a $(-1)^{F}$ symmetry, and we must say more about the algebra of the $\mathrm{T}$ symmetry in the correspondence. For spin systems, there are two universal possibilities labeled $\operatorname{pin}^{ \pm}$according to the implied structure group on spacetime, ${ }^{3}$

$$
\operatorname{pin}^{+} \Rightarrow \mathrm{T}^{2}=(-1)^{F}, \quad \operatorname{pin}^{-} \Rightarrow \mathrm{T}^{2}=1 .
$$

As we review in Appendix, the precise map between $\mathbb{Z}_{2}$ anomalies in fermionic theories and $\mathrm{T}$ anomalies holds when the latter is part of a pin ${ }^{-}$symmetry group.

In particular in Sec. IV, we apply these ideas to systems of free fermions in $(1+1) d$. There, as we review, the effect of interactions reduces the anomaly to a $\mathbb{Z}_{8}$ effect [26-30]. The correspondence (1.1) then implies the sharp result that the $(0+1) d$ fermion systems studied in [26,31] also have mod eight periodicity. Indeed, as we review, the ground state degeneracy of eight fermions with pin $^{-}$symmetry can be removed by a $\mathrm{T}$-invariant interaction.

\section{Further extensions}

Although we have focused above on $(1+1) d$ QFTs with $\mathbb{Z}_{2}$ symmetry, the correspondence (1.1) holds for general dimensions. As an illustration of this, in Sec. V, we consider $(3+1) d$ bosonic systems with $\mathbb{Z}_{2}$ unitary global symmetry. These theories have anomalies classified by the group $\mathbb{Z}_{2} \times \mathbb{Z}_{2}$ and have recently been discussed in [32]. As predicted by the correspondence (1.1), the classification of $\mathbf{T}$ anomalies in $(2+1) d$ is also $\mathbb{Z}_{2} \times \mathbb{Z}_{2}$ [2].

\footnotetext{
${ }^{3}$ In certain theories with additional global symmetry there can be other possible algebras obeyed by T; see, e.g., Ref. [25]. However, these do not play a role in the correspondence (1.1).
} 
We construct discrete gauge theories in $(3+1) d$ carrying these anomalies and explain how the $(2+1) d$ symmetry defect is decorated by an appropriate topological field theory with anomalous $\mathrm{T}$ symmetry where the lines can be either fermions or Kramers doublets.

\section{BOSONIC $\mathbb{Z}_{2}$ SYMMETRY IN $(1+1) d$}

In this section we investigate the correspondence (1.1) in the simplest nontrivial class of examples: $(1+1) d$ systems with $\mathbb{Z}_{2}$ global symmetry. We show that the defect Hilbert space $\mathcal{H}_{\mathcal{L}}$ for an anomalous $\mathbb{Z}_{2}$ line has Kramers degeneracy due to a time-reversal anomaly.

\section{A. Symmetry lines and the defect Hilbert space}

We start by reviewing some basic properties of the topological defect lines $\mathcal{L}$ implementing a unitary $\mathbb{Z}_{2}$ global symmetry in $(1+1) d$. A more detailed discussion can be found in [24]. Related ideas have been extensively discussed in [14-23].

A global symmetry in $(1+1) d$ is implemented by a topological defect line $\mathcal{L}$. The defining property of a topological defect line is that all physical observables such as correlation functions are invariant under a small deformation of the locus of $\mathcal{L}$. When the topological line is swept past a local operator $\phi(x)$, the correlation function is changed by the symmetry action of $\phi(x)$. For example, as we sweep a $\mathbb{Z}_{2}$ line past a $\mathbb{Z}_{2}$ even/odd local operator $\phi(x)$, the correlation function changes by \pm 1 . (See Fig. 1.)

Consider the theory on a cylinder $S^{1} \times \mathbb{R}$ with $\mathcal{L}$ running along the time $\mathbb{R}$ direction (see Fig. 4). The topological line $\mathcal{L}$ intersects with the spatial $S^{1}$, and therefore modifies the quantization by a twisted periodic boundary condition. This defines a defect Hilbert space denoted by $\mathcal{H}_{\mathcal{L}}{ }^{4}$ This is a different Hilbert space than the Hilbert space $\mathcal{H}$ without the $\mathbb{Z}_{2}$ line.

In the special case of conformal field theory, a state in the usual Hilbert space $\mathcal{H}$ is mapped to a local operator via the operator-state correspondence. A defect Hilbert space state $|\psi\rangle \in \mathcal{H}_{\mathcal{L}}$, on the other hand, is mapped to a nonlocal pointlike operator living at the end of the $\mathbb{Z}_{2}$ line. Since the topological line commutes with the stress tensor, the states in the defect Hilbert space $\mathcal{H}_{\mathcal{L}}$ are organized into representations of the left and right Virasoro algebras. In particular, the defect Hilbert space states can be diagonalized to have definite conformal weights $(h, \bar{h})$. In general, a state in $\mathcal{H}$ has integer spin $s=h-\bar{h}$, whereas as we will see below, a state in the defect Hilbert space $\mathcal{H}_{\mathcal{L}}$ may have fractional spin [23,24] (see also [33]).

We can also consider the insertion of multiple symmetry lines extended along time. This defines a multidefect

\footnotetext{
${ }^{4}$ When the $\mathbb{Z}_{2}$ is nonanomalous, the $\mathbb{Z}_{2}$ even sector $\mathcal{H}_{\mathcal{L}}^{+}$of the defect Hilbert space $\mathcal{H}_{\mathcal{L}}$ before gauging is the twisted sector of the orbifold theory.
}

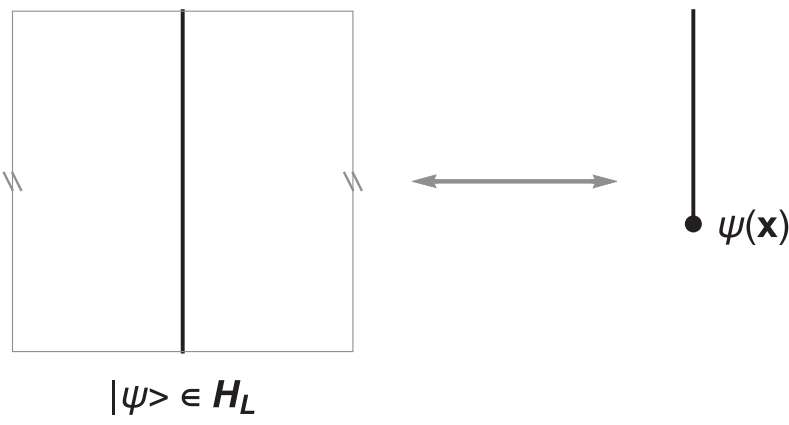

FIG. 4. The defect Hilbert space $\mathcal{H}_{\mathcal{L}}$ of a $\mathbb{Z}_{2}$ line quantized on a circle $S^{1}$. A state in the defect Hilbert space is mapped to an operator living at the end of the $\mathbb{Z}_{2}$ line via the operator-state correspondence.

Hilbert space $\mathcal{H}_{\mathcal{L L} \cdots \mathcal{L}}$. However, in the special case of $\mathbb{Z}_{2}$, we can fuse the symmetry lines pairwise to the trivial line. In particular, this means that the multidefect Hilbert spaces with an even number of $\mathbb{Z}_{2}$ symmetry lines are all isomorphic to the Hilbert space $\mathcal{H}$ of local operators, while those with an odd number of lines are all isomorphic to the defect Hilbert space $\mathcal{H}_{\mathcal{L}}$. This also means that the defect Hilbert space $\mathcal{H}_{\mathcal{L L}}$, which via the operator-state map are the operators living on the line, is isomorphic to the space $\mathcal{H}$ of ordinary local operators.

\section{B. $(1+1) d$ Bosonic $\mathbb{Z}_{2}$ anomaly}

The 't Hooft anomaly of a $\mathbb{Z}_{2}$ symmetry is encoded in a crossing relation of the topological symmetry lines. Consider a local patch of a general correlation function as in the gray circle on the left of Fig. 3. In this local patch we assume there are only two segments of $\mathbb{Z}_{2}$ lines, without other local operator insertions. Now imagine we replace the configuration in the local patch by the one on the right, without modifying the configuration outside the local gray patch. Notice that to continuously interpolate from one figure to another, we must pass through a singular configuration where the lines cross.

We would like to compare the two correlation functions, before and after the replacement. In a theory with a unique vacuum, the disk geometries in Fig. 3 define states on the circle with defect lines inserted and both states have $h=\bar{h}=0$. Therefore these two correlation functions can at most differ by a phase $\alpha$ [23,24]. Applying the same crossing rule twice, we conclude that $\alpha^{2}=1$. Hence $\alpha= \pm 1$.

The phase $\alpha$ is the 't Hooft anomaly of the $\mathbb{Z}_{2}$ global symmetry, which is classified by the group cohomology $H^{3}\left(\mathbb{Z}_{2}, U(1)\right)=\mathbb{Z}_{2}$. When $\alpha=+1$, the $\mathbb{Z}_{2}$ is nonanomalous, while when $\alpha=-1$, the $\mathbb{Z}_{2}$ is anomalous. One way to see this is to consider the torus partition function of the would-be $\mathbb{Z}_{2}$ orbifold theory. The orbifold partition function involves four terms with different $\mathbb{Z}_{2}$ line configurations, one of which is depicted in the center of Fig. 5. This configuration is generally ambiguous because of the 


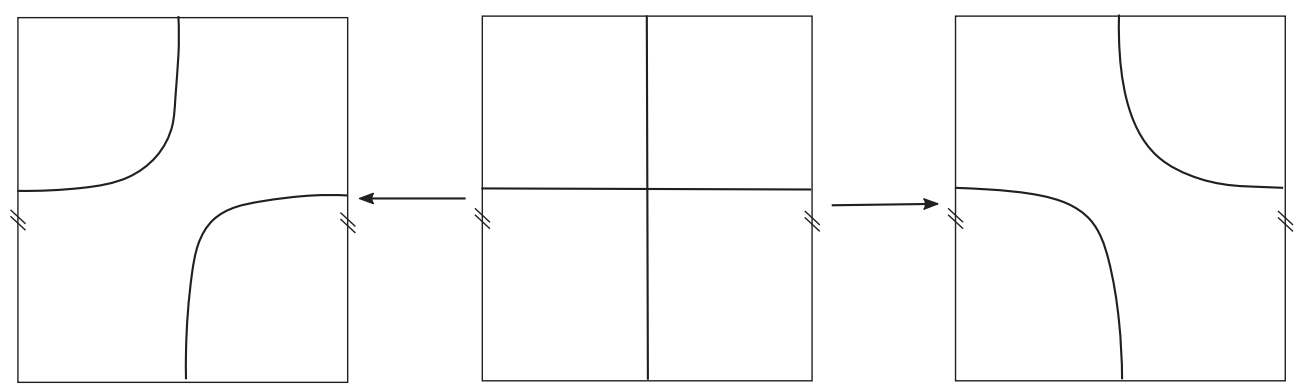

FIG. 5. When the $\mathbb{Z}_{2}$ is anomalous (i.e., $\alpha=-1$ ), the two resolutions (sides) of an intersection of lines (center) lead to different configurations.

intersection of the symmetry lines. The two possible resolutions of the intersection (shown at the two sides of Fig. 5) give the same answer if $\alpha=+1$, but not otherwise. Hence, the orbifold partition function is well-defined if $\alpha=+1$, but ambiguous and not modular invariant if $\alpha=-1$. In the latter case $\alpha=-1$, there is therefore an obstruction to gauging the $\mathbb{Z}_{2}$.

Another important consequence of the 't Hooft anomaly is a selection rule on the $\operatorname{spin} s=h-\bar{h}$ of the state in the defect Hilbert space. For a $\mathbb{Z}_{2}$ line, the spin of a state in $\mathcal{H}_{\mathcal{L}}$ is constrained to be $[23,24]$ (see also [33])

$$
s \in \begin{cases}\frac{\mathbb{Z}}{2}, & \text { if } \alpha=+1 \text { (nonanomalous), } \\ \frac{1}{4}+\frac{\mathbb{Z}}{2}, & \text { if } \alpha=-1 \text { (anomalous). }\end{cases}
$$

To argue for this result, we consider a $2 \pi$ rotation of a state in $\mathcal{H}_{\mathcal{L}}$ viewed in the operator picture of Fig. 6. By definition, this encodes the spin of the operator. By performing this operation twice and using the crossing relation of Fig. 3 we deduce that for all states in $\mathcal{H}_{\mathcal{L}}$, the spin obeys $\exp (4 \pi i s)=\alpha$, thus reproducing (2.1).

We can also see that the anomaly controls the expectation value of $\langle\mathcal{L}\rangle_{\mathbb{R}^{2}}$ of a closed loop of symmetry line in flat space. Indeed, using the crossing relation of Fig. 3, we obtain Fig. 7. And therefore $\langle\mathcal{L}\rangle_{\mathbb{R}^{2}}=\alpha$. We describe this equation in more detail in Sec. II C 2 below.

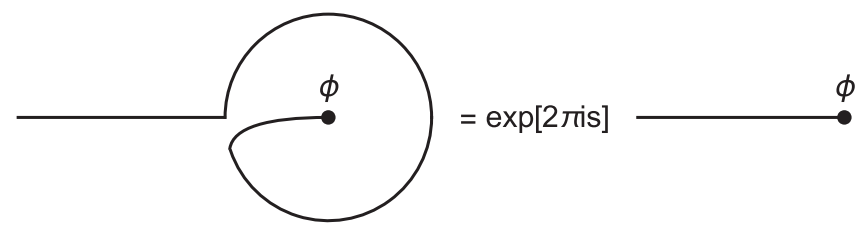

FIG. 6. The spin of an operator $\phi$ in $\mathcal{H}_{L}$ is captured by encircling the $\mathbb{Z}_{2}$ line around it as in the left figure.
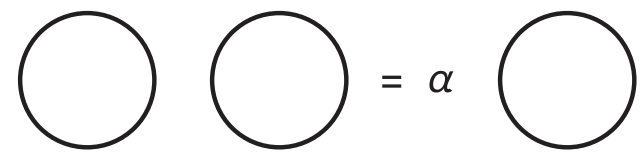

FIG. 7. Crossing relation between the $\mathbb{Z}_{2}$ lines.

\section{Orientation-reversal anomaly on the symmetry line}

We now discuss time-reversal symmetry on the line $\mathcal{L}$. As we will show, the $\mathcal{L}$ has a T anomaly if and only if the bulk has the $\mathbb{Z}_{2}$ anomaly. As a preliminary, we must first discuss why the line $\mathcal{L}$ has T symmetry to begin with. Since $\mathrm{T}$ symmetry acts geometrically by reversing orientation, this means we must discuss the orientation of the symmetry lines.

In general when defining correlation functions in QFTs with global symmetry, the associated symmetry defects must be oriented. Let $\mathcal{L}$ denote such an oriented defect, and $\overline{\mathcal{L}}$ the defect with opposite orientation. The meaning of the choice of orientation is that if $\mathcal{L}$ is associated with the symmetry group element $g$, then $\overline{\mathcal{L}}$ is associated with the symmetry group element $g^{-1}$. From this point of view, the reason why $\mathbb{Z}_{2}$ symmetry is special is that the nontrivial element is its own inverse. Therefore, up to anomalies discussed below, there is no dependence on the orientation of $\mathcal{L}$; i.e., for $\mathbb{Z}_{2}$ lines $\mathcal{L} \cong \overline{\mathcal{L}}$ (on flat spacetime). This in essence is what defines the T symmetry of the defect $\mathcal{L}$.

Intuitively, we can then view the result (7) that $\langle\mathcal{L}\rangle_{\mathbb{R}^{2}}=$ $\alpha$ as a manifestation of a $T$ anomaly on $\mathcal{L}$. Indeed, orienting $\mathcal{L}$ and stretching it to be long and thin, we can view this result as stating that even for $\mathbb{Z}_{2}$, there can be phase ambiguities in comparing $\mathcal{L}$ and $\overline{\mathcal{L}}$. Our goal is now to make these remarks more precise.

\section{Symmetry lines on curved surfaces}

The most transparent way to define and detect a $T$ anomaly on the symmetry line $\mathcal{L}$ is to consider its properties on curved surfaces. We discuss these ideas following [23]. For now we let $\mathcal{L}$ be a symmetry line for a general symmetry group and subsequently specialize to the case of $\mathbb{Z}_{2}$.

As emphasized in the Introduction, on a flat manifold, the correlation functions of symmetry lines are invariant under any deformation of the line $\mathcal{L}$ as long as it does not pass through any local operators. What about on a curved orientable surface? In general in this situation, we should expect that the topological nature of the symmetry lines 


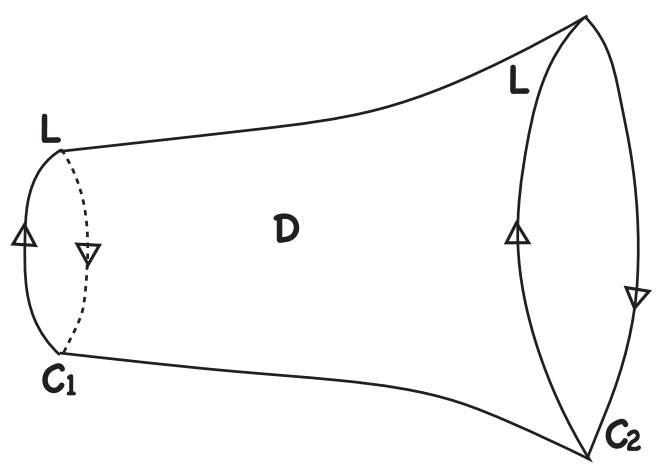

FIG. 8. The line $\mathcal{L}$, initially supported on an oriented curve $C_{1}$, is deformed past a domain $D$ to reach the final locus $C_{2}$.

may be modified by phases. This is because there may be a contact term in the operator product expansion (OPE) between the energy-momentum tensor and the topological line,

$$
T(x, y) \mathcal{L} \sim \theta_{\mathcal{L}} \delta^{\prime}(y),
$$

where the line is placed at $y=0$ and $\theta_{\mathcal{L}}$ is a constant that depends on the line. Equation (2.2) is fixed by (1) the righthand side only has support at $y=0$, and (2) it has mass dimension two. In the presence of a general metric, which acts as a source for $T$ this leads to phase modifications when $\mathcal{L}$ is deformed.

To see the consequences of (2.2), let the initial locus of the line $\mathcal{L}$ be an oriented curve $C_{1}$, and deform $\mathcal{L}$ past a domain $D$ to reach the final locus, another oriented curve $C_{2}$. This is illustrated in Fig. 8 .

The correlation functions are then modified by a phase ${ }^{5}$

$$
\exp \left[ \pm \frac{i \theta_{\mathcal{L}}}{4 \pi} \int_{D} d^{2} \sigma \sqrt{g} R\right]
$$

where the sign above is positive (negative) if $\partial D$ includes $C_{1}$ with positive (negative) orientation and $R$ is the Ricci scalar curvature on the surface. ${ }^{6}$ Alternatively we can also evaluate the effects of the same deformation using $\overline{\mathcal{L}}$ starting from $\bar{C}_{1}$ (the curve $C_{1}$ with opposite orientation), and ending at $\bar{C}_{2}$. Demanding that we arrive at the same phase implies that

$$
\theta_{\overline{\mathcal{L}}}=-\theta_{\mathcal{L}}
$$

The variation of the correlation function by the phase (2.3) has the appearance of an anomaly, i.e., a violation of

\footnotetext{
${ }^{5}$ We fix conventions so that the orientation of $D$ is the same as the ambient surface. The boundary of the domain $D$ is then either $\partial D=C_{1} \cup \bar{C}_{2}$ or $\partial D=\bar{C}_{1} \cup C_{2}$, depending on which direction we move the line on the surface.

${ }^{6}$ Our normalization is such that the Gauss-Bonnet formula reads $\int_{\Sigma} d^{2} \sigma \sqrt{g} R(g)=4 \pi \chi(\Sigma)$ with $\chi$ the Euler characteristic.
}

the topological nature of the lines by a phase. However, to check whether it is, in fact, meaningful, we must now determine if we can modify the definition of the symmetry line $\mathcal{L}$ by a suitable counterterm to remove this phase.

Indeed, phase (2.3) is not physical and can be removed using a counterterm precisely when $g \neq g^{-1}$. Specifically, we can redefine the line $\mathcal{L}$ by including

$$
\exp \left[\frac{i \phi_{\mathcal{L}}}{2 \pi} \int_{\mathcal{L}} d s K\right]
$$

where $K$ is the extrinsic curvature, normalized such that the counterclockwise integral along the boundary of a disk is $2 \pi$, and for consistency we define $\phi_{\overline{\mathcal{L}}}=-\phi_{\mathcal{L}}$. Applying the Gauss-Bonnet theorem to the cylindrical region $D$ that occurs in the calculation of (2.3) we have

$$
\frac{1}{4 \pi} \int_{D} d^{2} \sigma \sqrt{g} R(g)+\frac{1}{2 \pi} \int_{\partial D} d s K=\chi(D)=0,
$$

where the last equality follows from the fact that $D$ is an annulus. Thus, the phase (2.3) is canceled by the counterterm (2.5) if we choose $\phi_{\mathcal{L}}=\theta_{\mathcal{L}}$.

In particular, we note that while the phase (2.3) is nontrivial only on a curved manifold, the counterterm (2.5) is generally nonzero even on $\mathbb{R}^{2}$. (In this case the extrinsic curvature $K$ simply integrates to the total angle $2 \pi$ around any closed curve). Therefore the counterterm (2.5) changes the expectation value of a closed loop $\langle\mathcal{L}\rangle_{\mathbb{R}^{2}}$ by the arbitrary phase $\exp \left(i \phi_{\mathcal{L}}\right)$. Thus, when $g \neq g^{-1}$, the expectation value $\langle\mathcal{L}\rangle_{\mathbb{R}^{2}}$ does not have intrinsic meaning. ${ }^{7}$

\section{Orientation-reversal anomaly for $\mathbb{Z}_{2}$ lines}

Let us now turn to the special case of most interest, which is $\mathbb{Z}_{2}$ global symmetry. As we have discussed above, in this case locally, the symmetry line $\mathcal{L}$ is invariant under changes in orientation. This statement should be interpreted as an equality between $\mathcal{L}$ and $\overline{\mathcal{L}}$ on $\mathbb{R}^{2}$. In particular, this requirement forbids us from modifying the line by the extrinsic curvature counterterm (2.5). Thus, precisely for $\mathbb{Z}_{2}$ lines the expectation value $\langle\mathcal{L}\rangle_{\mathbb{R}^{2}}$ discussed in (7) is meaningful. ${ }^{8}$

\footnotetext{
${ }^{7}$ Mathematically, one can think of this extrinsic curvature counterterm as representing some of the exact elements that are set to zero in defining the group cohomology $H^{3}(G, U(1))$. Specifically, it represents those two-cochains of the form $\beta\left(g, g^{-1}\right) / \beta\left(g^{-1}, g\right)$ for $g$ in the zero-form group $G$.

${ }^{8}$ The negative expectation value of the $\mathbb{Z}_{2}$ line on $\mathbb{R}^{2}$ appears to be in tension with reflection positivity. If we had not insisted on the orientation-reversal symmetry $\mathcal{L}=\overline{\mathcal{L}}$ of a $\mathbb{Z}_{2}$ line on $\mathbb{R}^{2}$, then we have the freedom of adding the counterterm (2.5) to the line and modifying the expectation value to +1 . This tension between the reflection positivity and the orientation-reversal symmetry is similar to the one in $(2+1)$ dimensions discussed in Sec. 4.6 of [34]. We thank the referee for pointing out this analogy to us.
} 
Since the counterterm (2.5) is unavailable, the phase (2.3) is now an intrinsic physical property of the line. To interpret its effect we reexamine Fig. 8. The boundary of the cylindrical region $D$ is two isotopic lines with opposite orientation. Therefore, we can view the phase (2.3) as the cost of changing the orientation of the symmetry line $\mathcal{L}$ on a curved manifold. In particular, a nonzero phase $\theta_{\mathcal{L}}$ implies that on curved manifolds, in contrast to $\mathbb{R}^{2}$, the correlation functions of $\mathcal{L}$ depend on its orientation. This is an orientation-reversal or T anomaly on the defect $\mathcal{L}$.

We now show that this orientation reversal anomaly is precisely controlled by the bulk $\mathbb{Z}_{2}$ anomaly thus demonstrating the correspondence (1.1) in this case. First, note that the phase $\theta_{\mathcal{L}}$ is constrained by (7). Consider a small loop of $\mathcal{L}$ on a two-sphere. Near the north pole, the expectation value is approximated by $\langle\mathcal{L}\rangle_{\mathbb{R}^{2}}$, while near the south pole it is $\langle\overline{\mathcal{L}}\rangle_{\mathbb{R}^{2}}$. Deforming between them using the formula (2.3) we find

$$
\langle\mathcal{L}\rangle_{\mathbb{R}^{2}}=\exp \left( \pm 2 i \theta_{\mathcal{L}}\right)\langle\overline{\mathcal{L}}\rangle_{\mathbb{R}^{2}}
$$

Therefore, since $\langle\mathcal{L}\rangle_{\mathbb{R}^{2}}=\langle\overline{\mathcal{L}}\rangle_{\mathbb{R}^{2}}$ we deduce that $\theta_{\mathcal{L}}$ is either zero or $\pi$. In the former case there is no T anomaly on the line, while in the latter case there is a $\mathrm{T}$ anomaly.

Similarly, we can compare $\mathcal{L}$ wrapping the equator of the two-sphere to its behavior near a pole. On the equator, the expectation value looks locally like $\mathcal{L}$ wrapping the nontrivial cycle on the cylinder $S^{1} \times \mathbb{R}$. This expectation value is easy to determine. Since there is no operator insertion, we can view the defect $\mathcal{L}$ as acting on the vacuum in Hilbert space $\mathcal{H}$ of states on $S^{1}$. In any unitary theory, the vacuum on the circle is unique and symmetry preserving, and therefore $\langle\mathcal{L}\rangle_{S^{1} \times \mathbb{R}}=+1$. Therefore, deforming between the equator and a pole (see Fig. 9) and using the formula (2.3) we derive

$$
\langle\mathcal{L}\rangle_{\mathbb{R}^{2}}=\exp \left(i \theta_{\mathcal{L}}\right)\langle\mathcal{L}\rangle_{S^{1} \times \mathbb{R}}
$$

Combining (7) and the above, we conclude that In a unitary theory, the $\mathbb{Z}_{2}$ line $\mathcal{L}$ has the orientation-reversal $(\mathrm{T})$ anomaly if and only if the $\mathbb{Z}_{2}$ is anomalous, ${ }^{9}$

$$
\exp \left(i \theta_{\mathcal{L}}\right)=\alpha
$$

\footnotetext{
${ }^{9}$ This derivation requires unitarity. Indeed, in a nonunitary theory, the cylinder expectation value of a symmetry line may not be +1 . Then the above relation is modified to $\exp \left(i \theta_{\mathcal{L}}\right)=\frac{\alpha}{\langle\mathcal{L}\rangle_{S^{1} \times \mathbb{R}}}$.

For example, if $\langle\mathcal{L}\rangle_{S^{1} \times \mathbb{R}}=-1$ but $\alpha=-1$, then the anomalous $\mathbb{Z}_{2}$ line does not have the orientation-reversal anomaly in this nonunitary theory. Such an anomalous $\mathbb{Z}_{2}$ without an orientationreversal anomaly on the symmetry line can be found in, for example, the $(3,5)$ minimal model.
}

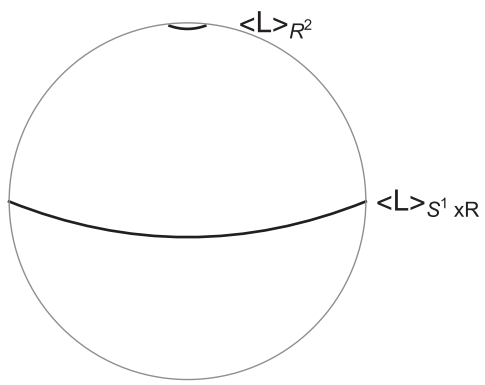

FIG. 9. The expectation value of an anomalous $\mathbb{Z}_{2}$ changes as we deform it on a curved manifold. For example, the vacuum expectation value near the north pole of a two-sphere differs from that around the equator by a sign.

\section{KRAMERS DOUBLETS IN THE DEFECT HILBERT SPACE}

We now illustrate the conclusion of the previous section in several examples. As discussed above, we interpret the orientation-reversal anomaly of an anomalous $\mathbb{Z}_{2}$ line as the time-reversal anomaly for the defect Hilbert space $\mathcal{H}_{\mathcal{L}}$. This gives a direct physical realization of the isomorphism (1.1) in the special case of $(1+1) d$ bosonic $\mathbb{Z}_{2}$ anomalies using the language of topological defect lines.

A $(0+1) d$ bosonic theory with $\mathrm{T}$ symmetry has an anomaly exactly when $T$ acts projectively, i.e., $\mathrm{T}^{2}=-1$ on $\mathcal{H}_{\mathcal{L}}$. This results in the Kramers doublet degeneracy in the defect Hilbert space $\mathcal{H}_{\mathcal{L}}$. Below we will demonstrate in examples that the defect Hilbert space $\mathcal{H}_{\mathcal{L}}$ has the Kramers degeneracy if and only if the bulk $\mathbb{Z}_{2}$ symmetry is anomalous. Most of our examples are diagonal rational CFTs, whose defect Hilbert space $\mathcal{H}_{\mathcal{L}}$ degeneracies are given by the fusion coefficients $N_{j k}^{i}$ [15] (see also [23]).

\section{A. $\widehat{\mathfrak{s t}(2)},{ }_{1}$ WZW model}

The $\widehat{\mathfrak{s i t}(2})_{1}$ Wess-Zumino-Witten (WZW) model has two current algebra primaries, the vacuum $|0,0\rangle$ and the four spin one-half primaries $\left|\frac{1}{4}, \frac{1}{4}\right\rangle_{ \pm, \pm}$of weight $h=\bar{h}=\frac{1}{4}$. The \pm stands for the $J_{0}^{3}$ and $\bar{J}_{0}^{3}$ eigenvalues. This theory has an anomalous $\mathbb{Z}_{2}$ global symmetry $(\alpha=-1)$ which commutes with the $\widehat{\mathfrak{s} \mathfrak{u}(2)} \times \widehat{\mathfrak{g} \mathfrak{\mathfrak { t }}(2)}$ current algebra and acts on the primaries as

$$
\mathbb{Z}_{2}:|0,0\rangle \mapsto|0,0\rangle, \quad\left|\frac{1}{4}, \frac{1}{4}\right\rangle_{ \pm, \pm} \mapsto-\left|\frac{1}{4}, \frac{1}{4}\right\rangle_{ \pm, \pm} .
$$

This $\mathbb{Z}_{2}$ can be thought of as the center of the left $\widehat{\mathfrak{s i t}(2)}$.

The defect Hilbert space $\mathcal{H}_{\mathcal{L}}$ of this anomalous $\mathbb{Z}_{2}$ has been studied, for example, in [24]. It consists of two current algebra primaries with $h=\frac{1}{4}$ and $\bar{h}=0$, and another two with $h=0$ and $\bar{h}=\frac{1}{4}$. Each one of them is a doublet under either the left or the right $\widehat{\mathfrak{s i t}(2)}$ current algebra: 


$$
\mathcal{H}_{\mathcal{L}}:\left|\frac{1}{4}, 0\right\rangle_{ \pm}, \quad\left|0, \frac{1}{4}\right\rangle_{ \pm} .
$$

Note that they have fractional spin $s=h-\bar{h}= \pm \frac{1}{4}$, consistent with the spin selection rule for states in $\mathcal{H}_{\mathcal{L}}(2.1)$. Moreover, we indeed see that there is a twofold degeneracy for every state of a given $(h, \bar{h})$ which is the expected Kramers degeneracy.

\section{B. $T^{2}$ CFT with no time-reversal symmetry}

In the general construction in Sec. II, the $(1+1) d$ theory is assumed to have a $\mathbb{Z}_{2}$ global symmetry, but is not in general time-reversal invariant. The $\widehat{\mathfrak{s t a}(2)}$, WZW model has time-reversal symmetry, and here we discuss an example without. ${ }^{10}$

The example we will consider is the free CFT with target space being a two-dimensional torus $T^{2}$, with a general metric and $B$ field. We will follow the convention in [35] with $\alpha^{\prime}=1$. The two scalar fields of the $T^{2} \mathrm{CFT}$ are normalized to have periodicities

$$
X^{1} \sim X^{1}+2 \pi R, \quad X^{2} \sim X^{2}+2 \pi R .
$$

The metric and the $B$ field of the $T^{2}$ CFT will be denoted as $G_{m n}$ and $B_{m n}$ with $m, n=1,2$, parametrizing the moduli space of the $T^{2}$ CFT. Since we have only two scalars, there is only one $B$ field, $B \equiv B_{12}$.

The metric moduli includes the overall volume and the complex structure moduli $\tau$. The overall volume can be parametrized by the $R$ above, while the complex structure $\tau$ is encoded in $G_{m n}$ as

$$
G_{m n}=\left(\begin{array}{cc}
1 & \tau_{1} \\
\tau_{1} & |\tau|^{2}
\end{array}\right)
$$

where $\tau=\tau_{1}+i \tau_{2}$ and $|\tau|^{2}=\tau_{1}^{2}+\tau_{2}^{2}$.

The spectrum of local operators can be described as follows. Let

$$
v_{m} \equiv \frac{n_{m}}{R}-B_{m n} w^{n} R,
$$

where $n_{m}, w^{n} \in \mathbb{Z}$ are the momentum and winding numbers. Next we define

$$
v_{L}^{m}=v^{m}+w^{m} R, \quad v_{R}^{m}=v^{m}-w^{m} R,
$$

\footnotetext{
${ }^{10}$ Another example is the tensor product of the $\widehat{\mathfrak{s t}(2)}, \mathrm{WZW}$ model with the holomorphic Monster CFT. This theory does not have $(1+1) d$ time-reversal symmetry, and it has an anomalous $\mathbb{Z}_{2}$ in the $\widehat{\mathfrak{s i t}(2)}$, WZW sector. Its defect Hilbert space is what we described in the previous section tensor product with the Monster CFT, which is not invariant under $h \leftrightarrow \bar{h}$ but still has the Kramers degeneracy in states with the same $h$ and $\bar{h}$.
}

where the indices are raised and lowered by $G_{m n}$ and $G^{m n}$. For example, $v^{m}=G^{m n} v_{n}$. The current algebra primaries in the $T^{2}$ CFT are labeled by four integers, $\left(n_{1}, n_{2}, w^{1}, w^{2}\right)$, with weights

$$
h=\frac{1}{4} G_{m n} v_{L}^{m} v_{L}^{n}, \quad \bar{h}=\frac{1}{4} G_{m n} v_{R}^{m} v_{R}^{n} .
$$

At a generic point on the moduli space with nontrivial $B$ field, the $(1+1) d$ theory does not have a time-reversal symmetry that exchanges $h$ and $\bar{h}$.

There are various anomalous $\mathbb{Z}_{2}$ symmetries. We will focus on the anomalous $\mathbb{Z}_{2}$ subgroup of $\operatorname{diag}\left(U(1)_{n_{1}} \times U(1)_{w^{1}}\right)$. The twisted sector operators are those with $n_{1}, w^{1} \in \frac{1}{2}+\mathbb{Z}$ and $n_{2}, w^{2} \in \mathbb{Z}$ (see, for example, $[24,36])$. We therefore find that the defect Hilbert space has a twofold Kramers degeneracy in states with the same $h$ and $\bar{h}$, which is realized by the action on the mode numbers

$\left(n_{1}, n_{2}, w^{1}, w^{2}\right) \rightarrow\left(-n_{1}-1,-n_{2},-w^{1}-1,-w^{2}\right)$.

\section{Ising model}

Finally, let us consider an example where the $(1+1) d$ $\mathbb{Z}_{2}$ symmetry is nonanomalous, and show that the defect Hilbert space has no Kramers degeneracy.

The $(1+1) d$ Ising model has three Virasoro primaries, the vacuum 1 with $h=\bar{h}=0$, the energy operator $\epsilon$ with $h=\bar{h}=\frac{1}{2}$, and the spin field $\sigma$ with $h=\bar{h}=\frac{1}{16}$. There is a $\mathbb{Z}_{2}$ symmetry that flips the sign of the spin field

$$
\mathbb{Z}_{2}: 1 \rightarrow 1, \quad \epsilon \rightarrow \epsilon, \quad \sigma \rightarrow-\sigma .
$$

The $\mathbb{Z}_{2}$ symmetry is nonanomalous $\alpha=+1$. Indeed, the Ising CFT is self-dual under the $\mathbb{Z}_{2}$ orbifold.

The defect Hilbert space $\mathcal{H}_{\mathcal{L}}$ of the $\mathbb{Z}_{2}$ line consists of following Virasoro primaries $|h, \bar{h}\rangle$ (see, for example, [24]):

$$
\bar{\psi}(\bar{z})=\left|0, \frac{1}{2}\right\rangle, \quad \psi(z)=\left|\frac{1}{2}, 0\right\rangle, \quad \mu(z, \bar{z})=\left|\frac{1}{16}, \frac{1}{16}\right\rangle .
$$

Under the operator-state correspondence, they are mapped to operators living at the end of the $\mathbb{Z}_{2}$ line. The $\left|0, \frac{1}{2}\right\rangle$ and $\left|\frac{1}{2}, 0\right\rangle$ states are the free Majorana fermions $\psi(z), \bar{\psi}(\bar{z})$ with half-integral spins. The $\left|\frac{1}{16}, \frac{1}{16}\right\rangle$ state in $\mathcal{H}_{\mathcal{L}}$ is the disorder operator $\mu(z, \bar{z})$, which is not mutually local with the spin field $\sigma(z, \bar{z})$ in $\mathcal{H}$ because the latter is $\mathbb{Z}_{2}$ odd. Note that the spins of these states are consistent with the spin selection rule (2.1). We see that there is no Kramers degeneracy for the states in $\mathcal{H}_{\mathcal{L}}$; hence there is no $\mathrm{T}$ anomaly in this case. 


\section{FERMIONIC EXAMPLES IN $(1+1) d$}

In this section we give an example illustrating the correspondence $(1.1)$ for spin theories in $(1+1) d$. As we review below, in this case possible anomalies for $\mathbb{Z}_{2}$ are classified by $\mathbb{Z}_{8}[4,26-30]$. Similarly, in $(0+1) d$, T anomalies for theories with $\mathrm{T}^{2}=1$ (i.e., pin $^{-}$structure) are also classified by $\mathbb{Z}_{8}[4,6,26,31] .{ }^{11}$ The correspondence (1.1) then directly relates these results. We concentrate our analysis on free fermion systems and their interactions.

\section{A. $\mathbb{Z}_{2}$ invariant $(1+1) d$ fermions}

Consider a $(1+1) d$ system of $N$ free (nonchiral) Majorana fermions $\chi^{i}(z, \bar{z})$. We will denote their leftand right-moving components as $\psi^{i}(z)$ and $\rho^{i}(\bar{z})$, respectively. We define the global $\mathbb{Z}_{2}$ symmetry of interest to be the left-moving fermion number $(-1)^{F_{L}}$ which acts on $\psi^{i}$, but under which the right moving fermions $\rho^{i}(\bar{z})$ are neutral. There is also the total fermion number symmetry that acts diagonally on both left- and right-moving fields. These $N$ fermions have an $S O(N) \times S O(N)$ global symmetry under which $\psi$ and $\rho$ transform, respectively, as $(v, 1)$ and $(1, v)$ where $v$ is the vector representation. Under the diagonal $S O(N) \subset S O(N) \times S O(N)$ the Majoranas $\chi^{i}$ are thus also in the vector representation.

The $(-1)^{F_{L}}$ symmetry prohibits quadratic mass terms $\psi^{i}(z) \rho^{j}(\bar{z})$, and hence, at the quadratic level, the number of fermions $N$ is protected. We now investigate to what extent this statement survives interactions. Consider a quartic interaction invariant under the diagonal $S O(N)$ :

$$
H_{\mathrm{int}}=q\left(\sum_{i=1}^{N} \bar{\chi}^{i} \chi^{i}\right)^{2} .
$$

Following [27-30], our goal is to see to when such interactions can lead to a trivially gapped state preserving the chiral symmetry. Our treatment closely parallels the review [38].

For two fermions, the first case where this interaction is possible, the free theory describes a $c=1$ model and the quartic fermion interaction is the unique exactly marginal operator. Therefore in this case the theory remains gapless for all $q$. For $N>2$, the operator (4.1) is marginal but not exactly marginal. For one sign of $q$ this leads again to the gapless free fermions; however, for the other sign of $q$, the interaction operator becomes important at long distances leading to the $S O(N)$ Gross-Neveu model [39]. This model is gapped and at a strong coupling scale $\Lambda$ generates an expectation value

\footnotetext{
${ }^{11}$ Mathematically, the $(1+1) d$ SPT associated with the Tinvariant fermions is the $\mathbb{Z}_{8}$-valued Arf-Brown-Kervarie cobordism invariant of pin $^{-}$two-manifolds. Meanwhile the $(2+1) d \mathbb{Z}_{2}$ SPT can be constructed by evaluating an $S O(N)_{1}$ Chern-Simons path integral coupled to a background magnetic field [37].
}

$$
\left\langle\sum_{i=1}^{N} \bar{\chi}^{i} \chi^{i}\right\rangle= \pm \Lambda
$$

This expectation value spontaneously breaks the $(-1)^{F_{L}}$ symmetry leading to two degenerate ground states.

Something special happens with eight fermions; however, that will enable us to find an appropriate interaction to gap the system. The special feature that we need is triality [40]. This allows us to present the theory of the eight fermions in dual description where there are again eight Majorana fermions $\tilde{\chi}^{m}(m=1, \ldots, 8)$ but now the left- and right-moving parts transform under $S O(8) \times S O(8)$ as $(s, 1)$ and $(1, s)$ where $s$ is an eight-dimensional spinor representation. These spinor fermions are coupled to a dynamical $\mathbb{Z}_{2} \times \mathbb{Z}_{2}$ gauge theory with gauge fields $x$ and $y$ coupling, respectively, to the $(-1)^{\tilde{F}}$ and $(-1)^{\tilde{F}_{L}}$ symmetries of the fermions $\tilde{\chi}^{m}$. Triality has an important interplay with operators in various sectors of the theory. In the $\chi$ theory, there are spin fields $\sigma^{m, n}$ that are $(R, R)$ sector operators of dimension $(1 / 2,1 / 2)$ and transform as $(s, s)$ under the $S O(8) \times S O(8)$ global symmetry. Crucially for our purposes, under triality these spin fields are exchanged with bilinears in the $\tilde{\chi}$ fermions. ${ }^{12}$

Since we are interested in gapping the model while preserving the original $(-1)^{F_{L}}$ symmetry, we should track how this behaves under the above triality. Let $a$ be the background gauge field for the $(-1)^{F_{L}}$. In terms of the original vector-valued fermion variables $\chi$, the coupling to $a$ appears in the kinetic term. However, in the dual theory with $S O(8)$ spinor fermions $\tilde{\chi}$, the symmetry $(-1)^{F_{L}}$ does not couple directly to the fermions but rather couples to the twisted sector operators that emerge from the dynamical $\mathbb{Z}_{2} \times \mathbb{Z}_{2}$ gauge fields $x$ and $y$. Specifically this means that $a$ enters the action for the dual theory as $\exp (i \pi a \cup(x+y))$, where $x, y, a \in H^{1}\left(\Sigma, \mathbb{Z}_{2}\right)$ and $\Sigma$ is spacetime.

We now return to our original goal of trivially gapping the theory of eight fermions $\chi$. An enlightening attempt, which ultimately will not succeed, is to try to add classically marginal potential which is invariant under the diagonal $S O(8) \subset S O(8) \times S O(8)$ constructed out of quadratic combination spin fields $\sigma^{m, n}$ transforming in the

\footnotetext{
${ }^{12}$ In fact, the existence of the triality shows that the chiral symmetry $(-1)^{\tilde{F}_{L}}$ of the $\tilde{\chi}$ theory, itself a theory of eight fermions, is nonanomalous in the sense that it can be gauged. Here we simply show how to use this triality to trivially gap the original model. To derive this triality from first principles, one can start with eight fermions in the vector representation of $S O(8)$, and sum over the spin structures (i.e., bosonization) to obtain the (bosonic) Spin (8), WZW model. The latter has a nonanomalous $\mathbb{Z}_{2} \times \mathbb{Z}_{2}$ global symmetry (see Appendix B of [24]). Next, the fermionizations (in the sense of $[36,41-44]$ ) of the $\operatorname{Spin}(8)_{1}$ WZW model with respect to the three order two elements give eight fermions in the vector, spinor, conjugate spinor representations of the $S O(8)$. Therefore, the triality can be realized as a composition of bosonization and fermionization.
} 
$(s, s)$. Using the triality discussed above, we can understand the effects of this interaction by mapping it to a quartic fermion operator in the dual fields $\tilde{\chi}$ :

$H_{\text {int }}=q\left(\sum_{m=1}^{8} \sigma^{m, m}\right)^{2} \leftrightarrow \tilde{H}_{\text {int }}=q\left(\sum_{m=1}^{8} \overline{\tilde{\chi}}^{m} \tilde{\chi}^{m}\right)^{2}$

From the right-hand side, it is clear that for one sign of $q$ we again flow to an $S O(8)$ Gross-Neveu model. Since the broken chiral symmetry $(-1)^{\tilde{F}_{L}}$ is gauged in the $\tilde{\chi}$ theory, this leads to a unique ground state in this sector. However, there remains the unbroken $(-1)^{\tilde{F}}$ symmetry which is also gauged and survives at long distances. Moreover, in the $\tilde{\chi}$ theory $a$ couples to the twisted sector of the surviving dynamical $\mathbb{Z}_{2}$ gauge field. Thus, the interaction (4.3) leads to two grounds states and again spontaneously breaks $(-1)^{F_{L}}$.

Finally, let us try an interaction preserving only the diagonal $\operatorname{Spin}(7)$,

$$
\begin{aligned}
H_{\mathrm{int}} & =q\left(\sum_{n=1}^{7} \sigma^{n, n}\right)^{2}+p\left(\sum_{n=1}^{7} \sigma^{n, n}\right) \sigma^{8,8} \\
\leftrightarrow & \tilde{H}_{\mathrm{int}}=q\left(\sum_{n=1}^{7} \overline{\tilde{\chi}}^{n} \tilde{\chi}^{n}\right)^{2}+p\left(\sum_{n=1}^{7} \overline{\tilde{\chi}}^{n} \tilde{\chi}^{n}\right) \overline{\tilde{\chi}}^{8} \tilde{\chi}^{8} .
\end{aligned}
$$

Working in the limit $|q| \gg|p|$ we first flow to a $\operatorname{Spin}(7)$ Gross-Neveu model. As above this leads to a unique ground state among these seven fermions. What remains at low energies is therefore the final fermion $\tilde{\chi}^{8}$ coupled to a dynamical $\mathbb{Z}_{2}$ gauge field $x$ acting on $\tilde{\chi}^{8}$ as the overall fermion number $(-1)^{F}$. Moreover, there is also a mass term arising from the chiral expectation value (4.2) and the interaction controlled by $p$. In summary, the long-distance effective action takes the form

$$
S_{\mathrm{eff}}=\int \overline{\tilde{\chi}}^{8} D_{x} \tilde{\chi}^{8}+m \overline{\tilde{\chi}}^{8} \tilde{\chi}^{8}+i \pi x \cup a .
$$

This is exactly the fermionic presentation of the $(1+1) d$ Ising model from gauging $(-1)^{F}$ of a nonchiral, massive Majorana fermion. In particular, the fermion mass operator is equivalent to the energy operator $\varepsilon$. For one sign of the mass, the theory spontaneously breaks the $(-1)^{F_{L}}$ symmetry. For the other sign, it is trivially gapped with a symmetric ground state as desired. ${ }^{13}$

\footnotetext{
${ }^{13}$ To recover the same result in the fermionic description, note that for one sign of the mass the low-energy limit of the fermions is trivial, leading to two ground states, while for the other sign of the mass, the fermion path-integral generates an $\operatorname{Arf}(x+s)$ interaction, where $x$ is the $\mathbb{Z}_{2}$ dynamical gauge field and $s$ is the underlying spin structure. This leads to a trivially gapped theory.
}

\section{B. T invariant $(0+1) d$ fermions}

Consider now a $(0+1) d$ system of $N$ free massless real fermions $\lambda^{i}(t)$. These fermions have a $\mathrm{T}$ symmetry that acts as

$$
\mathrm{T} \lambda^{i}(t) \mathrm{T}^{-1}=-\lambda^{i}(-t) \Rightarrow \mathrm{T}^{2}=+1
$$

We can try to add mass terms of the form $i m \lambda^{i} \lambda^{j}$ with real $m$ to gap out these fermions. However, these mass terms are forbidden by $\mathrm{T}$. Thus, including only quadratic mass terms, the number of fermions $N$ is protected.

To go further it is helpful to investigate the Hilbert space in more detail. For simplicity, we consider only even numbers of fermions and group them into complex pairs to define fermionic creation and annihilation operators:

$$
\begin{aligned}
& a_{n}=\frac{1}{\sqrt{2}}\left(\lambda^{2 n-1}+i \lambda^{2 n}\right), \\
& a_{n}^{\dagger}=\frac{1}{\sqrt{2}}\left(\lambda^{2 n-1}-i \lambda^{2 n}\right), \quad\left\{a_{n}, a_{m}^{\dagger}\right\}=\delta_{n m} .
\end{aligned}
$$

Each pair of fermions thus generates a Hilbert space spanned by states $|\downarrow\rangle$ and $|\uparrow\rangle$ :

$$
\begin{array}{lr}
a|\downarrow\rangle=0, & a^{\dagger}|\downarrow\rangle=|\uparrow\rangle, \\
a|\uparrow\rangle=|\downarrow\rangle, \quad a^{\dagger}|\uparrow\rangle=0 .
\end{array}
$$

$T$ acts on these states by $T|\downarrow\rangle=|\uparrow\rangle$ and $T|\uparrow\rangle=-|\downarrow\rangle$. Thus, the free system with $N$ fermions has a ground state degeneracy of $2^{N / 2}$.

Following [26], we now consider possible quartic fermion interactions to determine if we can find a system with a unique ground state. With four fermions we can add a $T$ invariant interaction term

$H_{\text {int }}=q \lambda^{1} \lambda^{2} \lambda^{3} \lambda^{4}=-q\left(a_{1}^{\dagger} a_{1}-\frac{1}{2}\right)\left(a_{2}^{\dagger} a_{2}-\frac{1}{2}\right)$.

Taking $q$ to be positive, we see that this term energetically penalizes states where the spins are not aligned. Therefore the fourfold ground state degeneracy is broken into a twofold degeneracy among the states $|\downarrow \downarrow\rangle$ and $|\uparrow \uparrow\rangle$. In particular, we still have ground state degeneracy. Similarly, one can argue that with six fermions we cannot find interactions to fully lift the degeneracy.

However, consider now eight fermions. We consider the interaction Hamiltonian

$$
\begin{aligned}
H_{\text {int }}= & q\left(\lambda^{1} \lambda^{2} \lambda^{3} \lambda^{4}+\lambda^{1} \lambda^{2} \lambda^{5} \lambda^{6}+\lambda^{1} \lambda^{2} \lambda^{7} \lambda^{8}+\lambda^{3} \lambda^{4} \lambda^{5} \lambda^{6}\right. \\
& \left.+\lambda^{3} \lambda^{4} \lambda^{7} \lambda^{8}+\lambda^{5} \lambda^{6} \lambda^{7} \lambda^{8}\right)-p \lambda^{1} \lambda^{3} \lambda^{5} \lambda^{7}
\end{aligned}
$$

We work in the limit where $q \gg p>0$. First neglecting $p$ and reasoning as above we deduce that there is an approximate ground state degeneracy among the two states 
where all spins are aligned $|\downarrow \downarrow \downarrow \downarrow\rangle$ and $|\uparrow \uparrow \uparrow \uparrow\rangle$. However now the $p$ interaction term acts to map between these two remaining states. This splits the degeneracy, leaving a unique $\mathrm{T}$ invariant ground state

$$
|\Omega\rangle=\frac{1}{\sqrt{2}}(|\downarrow \downarrow \downarrow \downarrow\rangle+|\uparrow \uparrow \uparrow \uparrow\rangle) .
$$

This shows that including interactions the number of fermions is only invariant modulo eight, and hence the possible $T$ anomaly is a mod eight effect.

\section{Twisted compactification}

As is clear from the previous sections both $(1+1) d \mathbb{Z}_{2}$ symmetric fermions and $(0+1) d \mathrm{~T}$ invariant fermions have a $\mathbb{Z}_{8}$ classification. But, the derivations of these two results appear somewhat different. The general correspondence (1.1) provides a direct map between them by considering the theory on the $\mathbb{Z}_{2}$ symmetry line.

Specifically, following our general discussion in Sec. I A, we can access the theory of the symmetry defect by a $\mathbb{Z}_{2}$ twisted circle compactification of the free $(1+1) d$ fermions. This twisted compactification of free fermions was also discussed in [28]. Since the $\mathbb{Z}_{2}$ acts chirally as $(-1)^{F_{L}}$ in the $(1+1) d$ free fermion theory, under this twisted compactification, the left-moving fermions $\psi^{i}(z)$ are periodic ( $\mathrm{R}$ sector), while the right-moving fermions $\rho^{i}(\bar{z})$ are antiperiodic (NS sector). In the low energy limit, we therefore obtain exactly $N(0+1) d$ Majorana fermions $\lambda^{i}(t)$ coming from the zero modes of the $(1+1) d$ left-moving fermions. In particular, this explains the fact that the classification of these systems, including interactions, coincide.

\section{AN EXAMPLE IN $(3+1) d$}

As a final example of the correspondence (1.1), we consider $(3+1) d$ bosonic theories with $\mathbb{Z}_{2}$ global symmetry. These possible anomalies for such theories have a $\mathbb{Z}_{2} \times \mathbb{Z}_{2}$ classification and have recently been discussed in the condensed matter literature in [32]. We can characterize both such anomalies by inflow from a five-dimensional bulk $M_{5}$. Let $a \in Z^{1}\left(M, \mathbb{Z}_{2}\right)$ denote the $\mathbb{Z}_{2}$ background cocycle field for the global symmetry. Then the bulk SPTs are given by the following classical actions:

$$
\begin{aligned}
& \exp \left(i \pi \int_{M_{5}} a \cup a \cup a \cup a \cup a\right) \text { and } \\
& \exp \left(i \pi \int_{M_{5}} a \cup w_{2} \cup w_{2}\right),
\end{aligned}
$$

where above $w_{2} \in Z^{2}\left(M_{5}, \mathbb{Z}_{2}\right)$ is the representative of the second Stiefel-Whitney class of $M_{5} \cdot{ }^{14}$ Mathematically, the

\footnotetext{
${ }^{14}$ The concrete representative of $w_{2}$ is given in [41].
}

first SPT above is captured by the group cohomology $H^{5}\left(\mathbb{Z}_{2}, U(1)\right) \cong \mathbb{Z}_{2}$, while the second involves the geometry nontrivially and goes beyond group cohomology.

We now consider $(2+1) d$ bosonic theories protected by time-reversal symmetry. As expected from the general isomorphism (1.1), the anomalies of such theories also admit a $\mathbb{Z}_{2} \times \mathbb{Z}_{2}$ classification [2]. We can realize the $(3+1) d$ SPTs for these anomalies by performing a twisted compactification of the SPTs in (5.1). Upon such a reduction, one factor of the gauge field $a$ is absorbed by the twisted circle direction, while the remaining factors each descend to the first Stiefel-Whitney class $w_{1}$ which can be viewed as a background gauge field for T symmetry (see Appendix). This leads to

$$
\begin{aligned}
& \exp \left(i \pi \int_{M_{4}} w_{1} \cup w_{1} \cup w_{1} \cup w_{1}\right) \text { and } \\
& \exp \left(i \pi \int_{M_{4}} w_{2} \cup w_{2}\right)
\end{aligned}
$$

which are indeed the correct $(2+1) d$ classical actions characterizing these SPTs.

\section{A. Discrete gauge theory construction}

Let us give examples of $(3+1) d$ theories coupled to a $\mathbb{Z}_{2}$ global symmetry realizing these anomalies. Consider, for instance, a version of $\mathbb{Z}_{2}$ gauge theory with dynamical gauge field $x_{1} \in C^{1}\left(M_{4}, \mathbb{Z}_{2}\right)$ and magnetic dual $y_{2} \in C^{2}\left(M_{4}, \mathbb{Z}_{2}\right) .{ }^{15}$ Here, $C^{p}\left(M_{4}, \mathbb{Z}_{2}\right)$ denotes the set of $\mathbb{Z}_{2}$-valued $p$-cochains on $M_{4}$. We couple this theory to the $\mathbb{Z}_{2}$ global symmetry through the action

$i \pi \int_{M_{4}}\left(x_{1} \cup \delta y_{2}+x_{1} \cup a \cup a \cup a+y_{2} \cup a \cup a\right)$.

Physically, one can think of these couplings as implying that the worldvolume theory of the dynamical extended operators in the theory (Wilson lines and surface operators) carry various anomalies for the $\mathbb{Z}_{2}$ global symmetry. For instance, $x_{1}$ has nontrivial holonomy surrounding a surface operator and the above leads to a cubic anomaly for the $\mathbb{Z}_{2}$ global symmetry inflowing onto this surface. It is straightforward to compute that this theory realizes the $a^{5}$ type anomaly described in (5.1).

Analogously, we can realize the $a w_{2}^{2}$ type anomaly by a version of $\mathbb{Z}_{2}$ gauge theory that is coupled to global symmetry as

\footnotetext{
${ }^{15}$ In the terminology of $[45,46], \mathbb{Z}_{2}$ gauge theory has an intrinsic 1 -form symmetry $\mathbb{Z}_{2}^{(1)}$ and an intrinsic 2-form symmetry $\mathbb{Z}_{2}^{(2)}$. If $A_{2}$ and $B_{3}$ are the associated background fields, the anomaly is $\exp \left(i \pi \int_{M_{5}} A_{2} \cup B_{3}\right)$. Below we couple the $\mathbb{Z}_{2} 0$-form global symmetry of interest to $\mathbb{Z}_{2}$ gauge theory through these higher-form symmetries.
} 


$$
i \pi \int_{M_{4}}\left(x_{1} \cup \delta y_{2}+x_{1} \cup a \cup w_{2}+y_{2} \cup w_{2}\right) .
$$

The couplings to $w_{2}$ now lead to worldvolume anomalies for the extended operators which involve their spin. For instance, $y_{2}$ has a nontrivial integral on a sphere surrounding a Wilson line and the coupling above then implies that in this theory such a line is a fermion.

In both theories (5.3) and (5.4) we can isolate the theory on the $\mathbb{Z}_{2}$ symmetry defect and exhibit the correspondence (1.1). Upon twisted compactification, in each case we find a sector that is a version of a $(2+1) d \mathbb{Z}_{2}$ gauge theory with dynamical fields $x_{1}$ and $y_{1}$ and modified quantum numbers for the line defects. Specifically, twisted compactification of (5.3) leads to

$i \pi \int_{M_{3}}\left(x_{1} \cup \delta y_{1}+x_{1} \cup w_{1} \cup w_{1}+y_{1} \cup w_{1} \cup w_{1}\right)$,

which has both Wilson lines for $x_{1}$ and $y_{1}$ as Kramers doublets realizing the $w_{1}^{4}$ anomaly in (5.2). Meanwhile twisted compactification of (5.4) results in

$$
i \pi \int_{M_{3}}\left(x_{1} \cup \delta y_{1}+x_{1} \cup w_{2}+y_{1} \cup w_{2}\right) .
$$

So in this case both Wilson lines for $x_{1}$ and $y_{1}$ are fermions living on the worldvolume of the bulk $\mathbb{Z}_{2}$ symmetry defect.

\section{ACKNOWLEDGMENTS}

We thank C.-M. Chang, P.-S. Hsin, Z. Komargodski, M. Levin, Y.-H. Lin, A. Neitzke, S. Ryu, N. Seiberg, Y. Tachikawa, J. Wang, and Y. Wang for interesting discussions. S. H. S. is supported by NSF Grant No. PHY1606531, the Roger Dashen Membership, the Simons Foundation/SFARI (651444, NS). F. Y. is supported by DOE Grant No. DE-SC0010008. This work benefited from the 2019 Pollica summer workshop, which was supported in part by the Simons Collaboration on the NonPerturbative Bootstrap and in part by the INFN.

Note added.-This work is submitted in coordination with [47], which contains partially overlapping results. We thank the authors of [47] for sharing their notes with us.

\section{APPENDIX: SMITH ISOMORPHISM BETWEEN COBORDISM GROUPS}

Here we summarize the mathematical formulation of the isomorphism (1.1) between the $\mathbb{Z}_{2}$ SPT phases in $d$ dimensions and time-reversal SPTs in $d-1$ dimensions, following $[4,11]$. For mathematics literature, see $[9,10]$.

We start with the isomorphism between interacting bosonic SPT phases. The $d$-dimensional interacting bosonic SPT phases with a unitary $\mathbb{Z}_{2}$ symmetry are classified by the following cobordism group $[2,4]$ :

$$
\Omega_{\mathrm{SO}}^{d}\left(B \mathbb{Z}_{2}\right):=\operatorname{Hom}\left(\Omega_{d}^{\mathrm{SO}}\left(B \mathbb{Z}_{2}\right), U(1)\right),
$$

where $\Omega_{d}^{\mathrm{SO}}\left(B \mathbb{Z}_{2}\right)$ is the bordism group of pairs $(M, a)$ of a $d$-dimensional oriented manifold $M$ and a $\mathbb{Z}_{2}$ background $a \in H^{1}\left(M, \mathbb{Z}_{2}\right)$ on $M$. The SPTs that can be made trivial after breaking the symmetry $G$ are classified by the reduced cobordism group

$$
\tilde{\Omega}_{\mathrm{SO}}^{d}\left(B \mathbb{Z}_{2}\right):=\operatorname{Hom}\left(\tilde{\Omega}_{d}^{\mathrm{SO}}\left(B \mathbb{Z}_{2}\right), U(1)\right),
$$

where the reduced bordism group $\tilde{\Omega}_{\mathrm{SO}}^{d}\left(\mathbb{Z}_{2}\right)$ is the quotient $\Omega_{\text {SO }}^{d}\left(\mathbb{Z}_{2}\right) / \Omega_{\text {SO }}^{d}(\mathrm{pt})$.

On the other hand, $(d-1)$-dimensional interacting fermionic SPT phases with a time-reversal symmetry T are classified by

$$
\Omega_{\mathrm{O}}^{d-1}(\mathrm{pt}):=\operatorname{Hom}\left(\Omega_{d-1}^{\mathrm{O}}(\mathrm{pt}), U(1)\right)
$$

where $\Omega_{d-1}^{\mathrm{O}}(\mathrm{pt})$ is the bordism group of $(d-1)$-dimensional unoriented manifolds to a point.

The isomorphism (1.1) relates the two bordism groups:

$$
f: \tilde{\Omega}_{d}^{\mathrm{SO}}\left(B \mathbb{Z}_{2}\right) \stackrel{\sim}{\rightarrow} \Omega_{d-1}^{\mathrm{O}}(\mathrm{pt}) .
$$

Concretely, given a bordism class $[M, a] \in \tilde{\Omega}_{d}^{\mathrm{SO}}\left(B \mathbb{Z}_{2}\right)$ with a representative pair $(M, a)$, its image under the isomorphism $f$ is the bordism class $[Y] \in \Omega_{d-1}^{\mathrm{O}}(\mathrm{pt})$ where $Y \subset M$ is a submanifold of $M$ that is Poincaré dual to $a{ }^{16}$ The isomorphism $f$ induces the correspondence between SPTs with $\mathbb{Z}_{2}$ symmetry and those with time-reversal symmetry:

$$
f^{*}: \Omega_{\mathrm{O}}^{d-1}(\mathrm{pt}) \stackrel{\sim}{\rightarrow} \tilde{\Omega}_{\mathrm{SO}}^{d}\left(B \mathbb{Z}_{2}\right)
$$

This direction of the isomorphism means that given a timereversal SPT in $d-1$ dimensions, one can construct a $d$-dimensional $\mathbb{Z}_{2}$ SPT by demanding that the $\mathbb{Z}_{2}$ symmetry defect supports the given time-reversal SPT. An explicit form of $f^{*}$ can be described as follows, generalizing the map discussed in Sec. I A. The theorem in $[48,49]$ asserts that any cobordism invariant in $\Omega_{\mathrm{O}}^{d-1}(\mathrm{pt})$ can be represented as a polynomial $P\left(w_{i}\right)$, where $w_{i}$ are the Stiefel-Whitney classes. Not all of the polynomials are independent of each other as there are relations among them. In particular, we have

\footnotetext{
${ }^{16}$ The Poincaré dual of $a \in H^{1}\left(M, \mathbb{Z}_{2}\right)$ can be taken to be a smooth submanifold. $a$ defines a classifying map $a: M \rightarrow \mathbb{R} \mathbb{P}^{k}$ with sufficiently large $k$, and the Poincaré dual can be taken to be the inverse image of $\mathbb{R} \mathbb{P}^{k-1} \subset \mathbb{R} \mathbb{P}^{k}$ under $a$. As generic $a$ is transverse to $\mathbb{R} \mathbb{P}^{k-1}, a^{-1}\left(\mathbb{R} \mathbb{P}^{k-1}\right)$ defines a smooth submanifold of $M$.
} 


$$
\begin{aligned}
\int_{M_{d}} w_{1}^{2 k+1} Q_{d-2 k-1}\left(w_{i \geq 2}\right) & =\int_{M_{d}} \beta\left(w_{1}^{2 k} Q_{d-2 k-1}\left(w_{i \geq 2}\right)\right) \\
& =\int_{M_{d}} w_{1}^{2 k} \beta\left(Q_{d-2 k-1}\left(w_{i \geq 2}\right)\right),
\end{aligned}
$$

where $M_{d}$ is a $d$-manifold, $Q_{d-2 k-1}\left(w_{i \geq 2}\right)$ is a degree$(d-2 k-1)$ polynomial of $w_{i \geq 2}$, and $\beta$ is the Bockstein operation. $\beta\left(Q_{d-2 k-1}\left(w_{i \geq 2}\right)\right)$ can be regarded as a degree$(d-2 k)$ polynomial of $w_{i \geq 3}$ because of the Wu formula. Therefore, without loss of generality, we can take the form of the representative $P\left(w_{i}\right) \in \Omega_{\mathrm{O}}^{d-1}(\mathrm{pt})$ to be

$$
P\left(w_{i}\right)=\sum_{k=0} w_{1}^{2 k} Q_{d-2 k-1}\left(w_{i \geq 2}\right)
$$

Similarly, a bordism invariant in $\Omega_{\mathrm{SO}}^{d}\left(B \mathbb{Z}_{2}\right)$ can be written as a polynomial $\tilde{P}$ of $\mathbb{Z}_{2}$ background $a$ and $w_{i \geq 2}$. Again, the polynomials are subject to relations. In particular, we have

$$
\begin{aligned}
\int_{M_{d}} a^{2 k} Q_{d-2 k}\left(w_{i \geq 2}\right) & =\int_{M_{d}}(\beta a) a^{2 k-2} Q_{d-2 k}\left(w_{i \geq 2}\right) \\
& =\int_{M_{d}} a^{2 k-1} \beta\left(Q_{d-2 k}\left(w_{i \geq 2}\right)\right),
\end{aligned}
$$

when $M_{d}$ is an oriented $d$-manifold. Then, without loss of generality, we can pick a representative $\tilde{P} \in \tilde{\Omega}_{\text {SO }}^{d}$ to have the form of

$$
\tilde{P}\left(a, w_{i \geq 2}\right)=\sum_{k=1} a^{2 k-1} Q_{d-2 k-1}\left(w_{i \geq 2}\right) .
$$

Now, the map (A5) is

$$
f^{*}: P\left(w_{1}, w_{i \geq 2}\right) \mapsto \tilde{P}\left(a, w_{i \geq 2}\right)=a \cup P\left(a, w_{i \geq 2}\right) .
$$

If we take the representatives in the cobordism classes to have the forms of (A7) and (A9), the explicit form of the map $f^{*}$ is

$$
f^{*}: \sum_{k=0} w_{1}^{2 k} Q_{d-2 k-1}\left(w_{i \geq 2}\right) \mapsto \sum_{k=0} a^{2 k+1} Q_{d-2 k-1}\left(w_{i \geq 2}\right) .
$$

The inverse of $f$,

$$
g=f^{-1}: \Omega_{d-1}^{\mathrm{O}}(\mathrm{pt}) \stackrel{\sim}{\rightarrow} \tilde{\Omega}_{d}^{\mathrm{SO}}\left(B \mathbb{Z}_{2}\right),
$$

can be constructed as follows. Given an unoriented $d-1$ dimensional manifold $Y$ representing a class of $\Omega_{d-1}^{\mathrm{O}}(\mathrm{pt})$ with the orientation (real line) bundle $\operatorname{det}(T Y)$, we construct the circle bundle $\tilde{M}_{Y}$ by taking the fiberwise unit circles of the direct sum $\operatorname{det}(T Y) \oplus \mathbb{R}$ of $\operatorname{det}(T Y)$ and the trivial real line bundle $\underline{\mathbb{R}}$. Further, we take the quotient of $\tilde{M}_{Y}$ by the $\mathbb{Z}_{2}$ acting on the fiber as a $\pi$ rotation and call it $M_{Y}$. The map $g$ is set to be $g([Y])=\left[\left(M_{Y}, a\right)\right]$, where $a$ is the $\mathbb{Z}_{2}$ connection corresponding to the $\mathbb{Z}_{2}$ bundle $\tilde{M}_{Y} \rightarrow M_{Y}$. The manifold $M_{Y}$ is oriented because when the transition function between patches of $Y$ is orientation reversing, it flips the orientation of the circle fiber by construction, so that it preserves the orientation of the total space. The Poincare dual of $a$ is by construction the base $Y$ of the bundle, and thus we have $f \circ g=\mathrm{id}$. For the proof of $g \circ f$, see [47].

The Pontryagin dual $g^{*}$ of (A12),

$$
g^{*}: \tilde{\Omega}_{\mathrm{SO}}^{d}\left(\mathrm{~B} \mathbb{Z}_{2}\right) \stackrel{\sim}{\rightarrow} \Omega_{\mathrm{O}}^{d-1}(\mathrm{pt})
$$

describes the $\mathbb{Z}_{2}$ twisted compactification of the given $\mathbb{Z}_{2}$ SPT on $M_{Y}$ to a time-reversal SPT on $Y$, which is the focus of the main text. In this construction, the time-reversal action on $Y$ involves the flipping of the circle fiber. This map $g^{*}$ can be expressed as

$g^{*}: \tilde{P}\left(a, w_{i \geq 2}\right) \mapsto P\left(w_{1}, w_{i \geq 2}\right)=\left.\partial_{a} \tilde{P}\left(a, w_{i \geq 2}\right)\right|_{a=w_{1}}$,

where $\partial_{a}$ is the formal partial derivative with respect to $a$. If we take the representatives in the cobordism classes to have the forms of (A7) and (A9), the explicit form of the map $g^{*}$ is

$$
g^{*}: \sum_{k=0} a_{1}^{2 k+1} Q_{d-2 k-1}\left(w_{i \geq 2}\right) \mapsto \sum_{k=0} w_{1}^{2 k} Q_{d-2 k-1}\left(w_{i \geq 2}\right),
$$

which is the inverse of (A11).

There is the generalization of the isomorphism between bosonic SPTs to the fermionic SPTs. ${ }^{17}$ Mathematically, the corresponding isomorphism between bordism groups is

$$
\tilde{\Omega}_{d}^{\mathrm{spin}}\left(B \mathbb{Z}_{2}\right) \stackrel{\sim}{\rightarrow} \Omega_{d-1}^{\mathrm{pin}^{-}}(\mathrm{pt}),
$$

where $\Omega_{*}^{\text {spin }}$ and $\Omega_{*}^{\text {pin }^{-}}$represent the cobordism groups of spin and pin $^{-}$manifolds. Pin ${ }^{-}$structure corresponds to the timereversal symmetry $T$ with $T^{2}=1$. Given a spin-manifold $M$ with a $\mathbb{Z}_{2}$ background $a$, we take the image of the manifolds as the Poincare dual $Y$ of $a$ as before. The spin structure of $M$ restricts onto the bundle $\left.T M\right|_{Y} \cong T Y \oplus N Y$, where $N Y$ is the normal bundle which is isomorphic to $\operatorname{det}(T Y) .{ }^{18}$ Now, a $\mathrm{pin}^{-}$structure on $Y$ is equivalent to a spin structure on

\footnotetext{
${ }^{17}$ There are further generalized versions of homomorphisms among different kinds of bordisms, which are discussed in $[4,10,11]$.

${ }^{18}$ As $\left.T M\right|_{Y}$ is oriented, we can take a local orthogonal frame so that the determinant of the transition function is 1 . Then $N Y \cong$ $\operatorname{det}(T Y)$ follows from the decomposition $\left.T M\right|_{Y} \cong T Y \oplus N Y$.
} 
$T Y \oplus \operatorname{det}(T Y)$, which can naturally be induced from the spin structure on $M$. Conversely, given a $\operatorname{pin}^{-}$structure on $Y$, it naturally extends to a spin structure on $\operatorname{det}(T Y) \oplus \underline{\mathbb{R}}$ and then can be restricted to a spin structure on $M_{Y}$. Therefore, the maps (A4) and (A12) are generalized to the case of (s)pin bordisms (A16).
[1] A. Kitaev, On the classification of short-range entangled states, in Seminar at Simons Center for Geometry and Physics, 2013.

[2] A. Kapustin, Symmetry protected topological phases, anomalies, and cobordisms: Beyond group cohomology, arXiv:1403.1467.

[3] A. Kapustin, Bosonic topological insulators and paramagnets: A view from cobordisms, arXiv:1404.6659.

[4] A. Kapustin, R. Thorngren, A. Turzillo, and Z. Wang, Fermionic symmetry protected topological phases and cobordisms, J. High Energy Phys. 12 (2015) 052.

[5] A. Kitaev, Homotopy-theoretic approach to SPT phases in action: Z16 classification of three-dimensional superconductors, in Seminar at UCLA (2015).

[6] D. S. Freed and M. J. Hopkins, Reflection positivity and invertible topological phases, arXiv:1604.06527.

[7] D. Gaiotto and T. Johnson-Freyd, Symmetry protected topological phases and generalized cohomology, J. High Energy Phys. 05 (2019) 007.

[8] K. Yonekura, On the cobordism classification of symmetry protected topological phases, Commun. Math. Phys. 368, 1121 (2019).

[9] A. Bahri and P. Gilkey, The eta invariant, pinc bordism, and equivariant spinc bordism for cyclic 2-groups, Pac. J. Math. 128, 1 (1987).

[10] P. B. Gilkey, The Geometry of Spherical Space Form Groups, 2nd ed. (World Scientific, Singapore, 2018) .

[11] Y. Tachikawa and K. Yonekura, Why are fractional charges of orientifolds compatible with Dirac quantization?, SciPost Phys. 7, 058 (2019).

[12] D. Gaiotto, A. Kapustin, N. Seiberg, and B. Willett, Generalized global symmetries, J. High Energy Phys. 02 (2015) 172.

[13] X. Chen, Y.-M. Lu, and A. Vishwanath, Symmetryprotected topological phases from decorated domain walls, Nat. Commun. 5, 3507 (2014),

[14] E. P. Verlinde, Fusion rules and modular transformations in 2D conformal field theory, Nucl. Phys. B300, 360 (1988).

[15] V. B. Petkova and J. B. Zuber, Generalized twisted partition functions, Phys. Lett. B 504, 157 (2001).

[16] J. Fuchs, I. Runkel, and C. Schweigert, TFT construction of RCFT correlators. 1. Partition functions, Nucl. Phys. B646, 353 (2002).

[17] J. Fuchs, I. Runkel, and C. Schweigert, TFT construction of RCFT correlators. 2. Unoriented world sheets, Nucl. Phys. B678, 511 (2004).

[18] J. Fuchs, I. Runkel, and C. Schweigert, TFT construction of RCFT correlators. 3. Simple currents, Nucl. Phys. B694, 277 (2004).
[19] J. Fuchs, I. Runkel, and C. Schweigert, TFT construction of RCFT correlators IV: Structure constants and correlation functions, Nucl. Phys. B715, 539 (2005).

[20] J. Fjelstad, J. Fuchs, I. Runkel, and C. Schweigert, TFT construction of RCFT correlators. V. Proof of modular invariance and factorisation, Theor. Appl. Categor. 16, 342 (2006), https://arxiv.org/abs/hep-th/0503194.

[21] A. Davydov, L. Kong, and I. Runkel, Invertible defects and isomorphisms of rational CFTs, Adv. Theor. Math. Phys. 15, 43 (2011).

[22] L. Bhardwaj and Y. Tachikawa, On finite symmetries and their gauging in two dimensions, J. High Energy Phys. 03 (2018) 189.

[23] C.-M. Chang, Y.-H. Lin, S.-H. Shao, Y. Wang, and $\mathrm{X}$. Yin, Topological defect lines and renormalization group flows in two dimensions, J. High Energy Phys. 01 (2019) 026.

[24] Y.-H. Lin and S.-H. Shao, Anomalies and bounds on charged operators, Phys. Rev. D 100, 025013 (2019).

[25] C. Córdova, P.-S. Hsin, and N. Seiberg, Time-reversal symmetry, anomalies, and dualities in $(2+1) d$, SciPost Phys. 5, 006 (2018).

[26] L. Fidkowski and A. Kitaev, Effects of interactions on the topological classification of free fermion systems, Phys. Rev. B 81, 134509 (2010).

[27] S. Ryu and S.-C. Zhang, Interacting topological phases and modular invariance, Phys. Rev. B 85, 245132 (2012).

[28] X.-L. Qi, A new class of $(2+1)$-dimensional topological superconductors with $\mathbb{Z}_{8}$ topological classification, New J. Phys. 15, 065002 (2013).

[29] H. Yao and S. Ryu, Interaction effect on topological classification of superconductors in two dimensions, Phys. Rev. B 88, 064507 (2013).

[30] Z.-C. Gu and M. Levin, Effect of interactions on twodimensional fermionic symmetry-protected topological phases with $Z_{2}$ symmetry, Phys. Rev. B 89, 201113 (2014).

[31] L. Fidkowski and A. Kitaev, Topological phases of fermions in one dimension, Phys. Rev. B 83, 075103 (2011).

[32] L. Fidkowski, J. Haah, M. B. Hastings, and N. Tantivasadakarn, Disentangling the generalized double Semion model, arXiv:1906.04188.

[33] L.-Y. Hung and X.-G. Wen, Universal symmetry-protected topological invariants for symmetry-protected topological states, Phys. Rev. B 89, 075121 (2014).

[34] E. Witten, Fermion path integrals and topological phases, Rev. Mod. Phys. 88, 035001 (2016).

[35] J. Polchinski, String Theory. Vol. 1: An Introduction to the Bosonic String, Cambridge Monographs on Mathematical Physics (Cambridge University Press, 2007). 
[36] W. Ji, S.-H. Shao, and X.-G. Wen, Topological transition on the conformal manifold, arXiv:1909.01425 [Phys. Rev. Research (to be published)].

[37] C. Córdova, P.-S. Hsin, and N. Seiberg, Global symmetries, counterterms, and duality in Chern-Simons matter theories with orthogonal gauge groups, SciPost Phys. 4, 021 (2018).

[38] D. Tong and C. Turner, Notes on 8 Majorana fermions, SciPost Phys. Lect. Notes 14 (2020).

[39] D. J. Gross and A. Neveu, Dynamical symmetry breaking in asymptotically free field theories, Phys. Rev. D 10, 3235 (1974).

[40] E. Witten, $D=10$ superstring theory, Prog. Math. Phys. 9, 395 (1983).

[41] D. Gaiotto and A. Kapustin, Spin TQFTs and fermionic phases of matter, Int. J. Mod. Phys. A 31, 1645044 (2016).

[42] A. Kapustin and R. Thorngren, Fermionic SPT phases in higher dimensions and bosonization, J. High Energy Phys. 10 (2017) 080.
[43] R. Thorngren, Anomalies and bosonization, arXiv:1810.04414.

[44] A. Karch, D. Tong, and C. Turner, A web of $2 d$ dualities: $\mathbf{Z}_{2}$ gauge fields and arf invariants, SciPost Phys. 7, 007 (2019).

[45] F. Benini, C. Córdova, and P.-S. Hsin, On 2-group global symmetries and their anomalies, J. High Energy Phys. 03 (2019) 118.

[46] C. Córdova and K. Ohmori, Anomalies, topological field theories, and symmetry-enforced gaplessness (to be published).

[47] I. Hason, Z. Komargodski, and R. Thorngren, Anomaly matching in the symmetry broken phase: Domain walls, $C P T$, and the smith isomorphism, SciPost Phys. 8, 062 (2020).

[48] R. Thom, Quelques propriétés globales des variétés différentiables, Commentarii mathematici Helvetici 28, 17 (1954).

[49] E. H. Brown, Jr. and F. P. Peterson, Relations among characteristic classes I, Topology 3, 39 (1964). 